\title{
A novel staging system and clinical predictive nomogram for more accurate staging and prognosis of malignant pancreatic intraductal papillary mucinous neoplasms: a population-based study
}

Haoxiang Zhang ${ }^{\dagger}$, Chenggang Gao ${ }^{\dagger}$, Jiaoshun Chen, Shihong Wu, Jianwei Bai and Tao Yin ${ }^{*}$

\begin{abstract}
Background: The current guidelines of the American Joint Committee on Cancer (AJCC) for the staging of exocrine pancreatic tumors seem inapplicable to malignant pancreatic intraductal papillary mucinous neoplasms (IPMN). Therefore, we aimed to improve the accuracy of clinical staging and prognosis for malignant IPMN by modifiing current AJCC system.

Methods: We extracted data of 2001 patients with malignant IPMN from the Surveillance, Epidemiology, and End Results database between 2000 and 2016. Of these, 1401 patients were assigned to the primary cohort and 600 patients to the validation cohort.

Results: In Kaplan-Meier analysis of the primary cohort, the current AJCC guidelines were unable to distinguish between certain tumor substages (IA and IB in the 7th, IB and IIA in the 8th). The modified system that we regrouped based on the median overall survival and hazard ratios, was superior in tumor stage classifications. Age $>70$ years, tumors located in the body or tail, high-grade differentiated tumors, surgery, chemotherapy, and tumor, lymph node, and metastasis (TNM) stage were identified as independent predictive factors for overall survival. Compared to that of TNM-based systems, the concordance index of the clinical predictive nomogram significantly improved $(0.819 ; 95 \%$ confidence interval, 0.805-0.833), with excellent area under the receiver operating characteristic curves (1-, 3-, and 5-year: $0.881,0.889$, and 0.879 , respectively). The calibration curves also showed good agreement between prediction and actual observation. The analysis of treatment modalities revealed that surgery resulted in better survival for all resectable malignant IPMN. The analysis of chemotherapy data reveals its potential in improving the prognosis of treatment for patients with locally advanced or distant metastases.
\end{abstract}

Conclusions: Our modified staging system improves the distinction of tumor stages. The nomogram was a more accurate and clinically reliable tool for prognosis prediction of patients with malignant IPMN.

\footnotetext{
*Correspondence: ytwhun@hust.edu.cn

${ }^{\dagger}$ Haoxiang Zhang and Chenggang Gao contributed equally to this manuscript

Department of Pancreatic Surgery, Union Hospital, Tongji Medical College, Huazhong University of Science and Technology, Wuhan 430022, China
}

(c) The Author(s) 2021. Open Access This article is licensed under a Creative Commons Attribution 4.0 International License, which permits use, sharing, adaptation, distribution and reproduction in any medium or format, as long as you give appropriate credit to the original author(s) and the source, provide a link to the Creative Commons licence, and indicate if changes were made. The images or other third party material in this article are included in the article's Creative Commons licence, unless indicated otherwise in a credit line to the material. If material is not included in the article's Creative Commons licence and your intended use is not permitted by statutory regulation or exceeds the permitted use, you will need to obtain permission directly from the copyright holder. To view a copy of this licence, visit http://creativecommons.org/licenses/by/4.0/. The Creative Commons Public Domain Dedication waiver (http://creativeco mmons.org/publicdomain/zero/1.0/) applies to the data made available in this article, unless otherwise stated in a credit line to the data. 
Keywords: American Joint Committee on Cancer, Clinical predictive nomogram, Pancreatic intraductal neoplasms, Survival

\section{Background}

Production of abnormally viscous mucus is a characteristic of pancreatic intraductal papillary mucinous neoplasms (IPMN). Since their first description in 1987, these rare tumors have been increasingly recognized [1]. The prevalence of IPMN is about 26 per 100,000 people; however, they are more common in the elderly, with an incidence of 99 per 100,000 people in those over the age of $60[2,3]$. IPMN are premalignant lesions that may progress to pancreatic ductal adenocarcinoma (PDAC), and this may take several years [4]. IPMN require either surveillance or surgical resection. As stated in the histological criteria of the World Health Organization, IPMN can be classified into benign and malignant tumors. Malignant tumors can be further subdivided into highgrade dysplasia (HGD) and invasive IPMN $[5,6]$. Compared to HGD (carcinoma in situ), invasive IPMN has a worse prognosis [7]. An accurate and comprehensive prognosis evaluation is particularly important. According to the 2018 European evidence-based guidelines, patients with main ductal IPMN should undergo resection [8]. All IPMN patients with jaundice, positive cytology findings, a solid component or an enhancing mural nodule measuring over $5 \mathrm{~mm}$, or a main pancreatic duct measuring over $10 \mathrm{~mm}$ in diameter have a high risk of malignancy, and surgical excision is recommended. Surgery remains the only potentially curative treatment for malignant IPMN, and there is scope for early detection and surgical cure $[6,8]$.

As the most acknowledged assessment staging system for tumors, the updated American Joint Committee on Cancer (AJCC), 8th edition staging system (AJCC 8th) for exocrine pancreatic tumors has been applied clinically since 2018 [9]. Its distinction from the AJCC 7th edition staging system (AJCC 7th) lies mainly in two aspects [10]. First, because of the difficulties in determining extrapancreatic extension clinically, the definitions of T2 $(>2 \mathrm{~cm}$ and $\leq 4 \mathrm{~cm}$ in the widest diameter) and T3 $(>4 \mathrm{~cm}$ in the widest diameter) are now based on the criteria for invasive tumors. Second, category N is subdivided into N0 (0 regional lymph nodes are positive), $\mathrm{N} 1$ (one to three regional lymph nodes are positive), and N2 (four or more regional lymph nodes are positive). Minor change includes the subcategorization of T1 into $\mathrm{T} 1 \mathrm{a}, \mathrm{T} 1 \mathrm{~b}$, and T1c based on size. Additionally, resectability was removed from the definition of T4 (Table 1).

Table 1 Definitions of AJCC TNM System

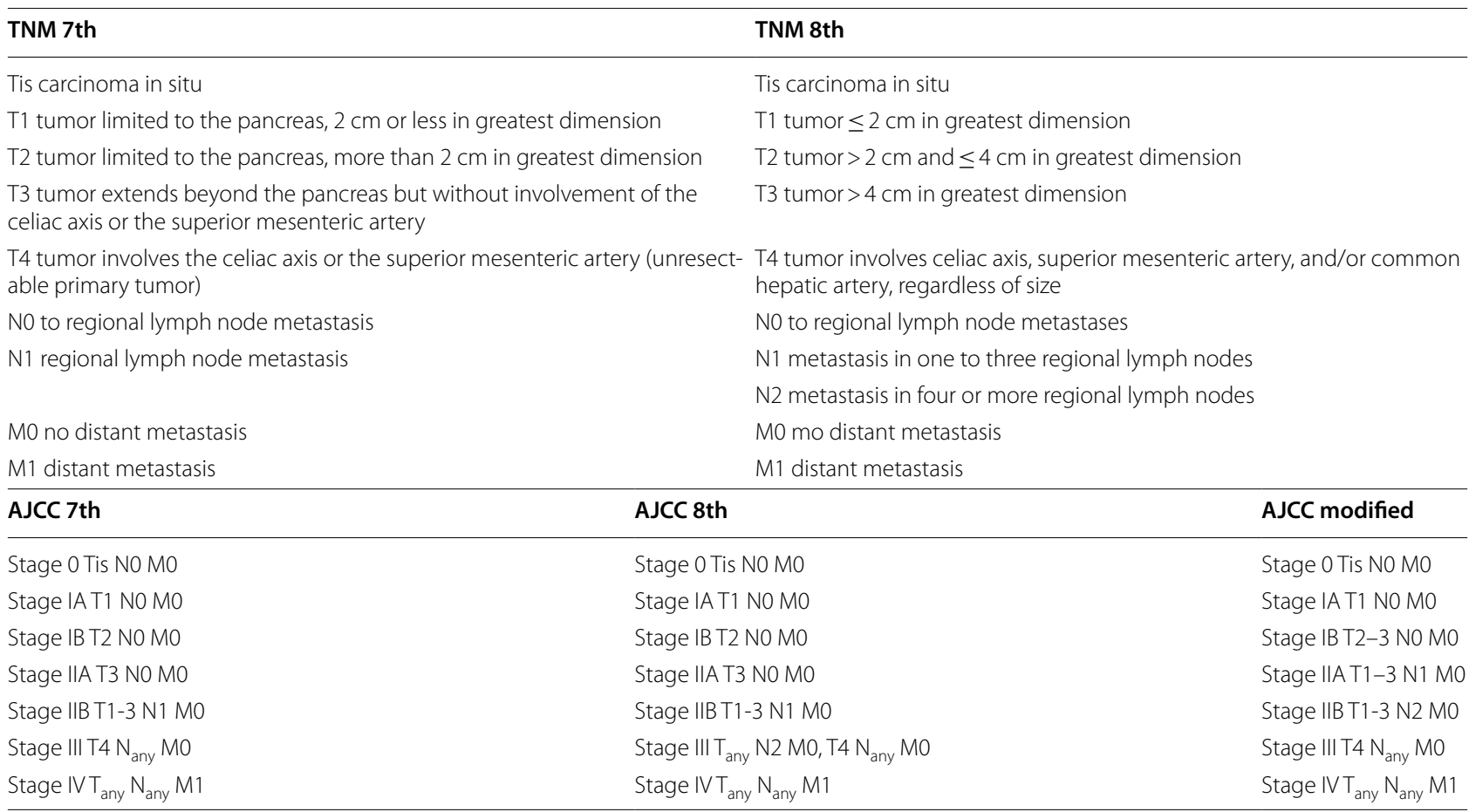


However, it has been stated that AJCC 8th is not applicable to the resection of PDAC, which accounts for $90 \%$ of pancreatic cancers [11].

Our study included patients with HGD and invasive IPMN for a more comprehensive overview of malignant IPMN. We aimed to improve the predictive accuracy of current staging systems using the Surveillance, Epidemiology, and End Results (SEER) database. We modified a novel AJCC-based system to improve the distinction of tumor stages and examined an extensive series of patients with malignant IPMN to investigate predictive factors, and develop a nomogram for a more precise prediction of the prognosis of malignant IPMN.

\section{Methods}

\section{Patients and data collection}

This retrospective data analysis of a cohort of patients, pathologically diagnosed with malignant IPMN from the SEER database (https://seer.cancer.gov/data-software/) between 2000 and 2016, was performed using the SEER* Stats software, version 8.3.6.1 (National Cancer Institute, Rockville, MD, US). Cases were selected based on their histology, which was identified using histology codes $(8050,8260,8450,8453,8471,8480,8481$, and 8503) and ICD-O-3 topography codes (C25.0-C25.9) [12]. The tumor stages (AJCC 7th and AJCC 8th) were derived using data on tumor size and invasion, lymph node involvement, and metastasis, all of which were available in the SEER database. Data on therapy including surgery, chemotherapy, and radiotherapy were also collected and analyzed. Patients who met the following criteria were included: (1) histology or puncture cytology positive for malignant IPMN (including HGD and invasive IPMN), (2) sufficient information to allow restaging according to current AJCC guidelines (7th and 8th), and (3) age $>20$ years and complete clinical and follow-up data. Patients in whom the above criteria were missing, were excluded. The definitions of and differences between AJCC 7th and AJCC 8th are shown in Table 1 and Fig. 1.

All patient data included no identifiable patient information and were accessed from the SEER database with permission. The study design was approved by the ethics committee of Union Hospital, Tongji Medical College, Huazhong University of Science and Technology, and the need for informed consent was waived owing to the study being a population study deemed not to constitute human subject research.

\section{Statistical analysis}

The study population was divided into a primary cohort and a validation cohort at a ratio of $7: 3$, using the caret package of R, version 4.0.3 (http://www.r-project.org/). Survival was calculated from the date of final diagnosis until the last follow-up or death and was analyzed using Kaplan-Meier curves. Cox proportional hazards regression was used for univariate and multivariate analyses. All predictors shown to be significant in the univariate analysis were investigated using multivariate analysis. Hazard ratios (HRs) and 95\% confidence intervals (CI) were analyzed. Significance was determined using logrank tests. The concordance index (C-index) and survival curves with pairwise comparison results by the logrank test was used to evaluate the discriminatory powers of the two different staging systems. A nomogram was constructed using the rms package within $R$, which included all significant independent factors in the multivariate analysis for predicting 1-, 3-, and 5-year overall survival (OS). The nomogram performance was assessed using the receiver operating characteristic (ROC) curves, C-index, and calibration curves. During the validation, the total points were calculated according to the established nomogram. Consecutively, Cox regression was performed. ROC curves, calibration curves, and C-index were derived based on the regression analysis [13].

All statistical tests were performed using the statistical language $\mathrm{R}$, version 4.0.3. All tests were two-sided, and a $\mathrm{p}$-value of $<0.05$ was considered statistically significant.

\section{Results}

\section{Baseline characteristics}

A total of 2001 patients with malignant IPMN from 2000 to 2006 were enrolled in this study, of which 1401 patients were included in the primary cohort, and 600 patients constituted the validation cohort. The baseline characteristics of patients in both cohorts are shown in Table 2. In the primary cohort, the median age of patients at diagnosis was 67 years. The male-to-female ratio was similar between the cohorts. The median OS of patients was 18 months (1-year survival rate, 58.4\%; 3-year survival rate, 35.9\%; 5-year survival rate, 29.3\%).

The major difference between AJCC 7th and AJCC 8th lay in the IA, IB, IIA, IIB, and III stages. In the primary cohort, $7.00 \%, 7.14 \%, 10.06 \%, 18.56 \%$, and $9.85 \%$ of patients were in stages IA, IB, IIA, IIB, and III, respectively, when using AJCC 7th. In contrast, according to AJCC 8th, 8.07\%, 7.64\%, 8.49\%, $11.99 \%$, and $16.42 \%$ of patients were in IA, IB, IIA, IIB, and III stages, respectively.

\section{Predictive prediction of current stage systems and stage modification}

In the primary cohort, the C-index using AJCC 7th and that with AJCC 8th were 0.779 (95\% CI 0.755-0.803) and 0.777 (95\% CI $0.753-0.801$ ), respectively. Further pairwise comparison by the log-rank test showed that stages IA and IB when using AJCC 7th and stages IB and IIA 


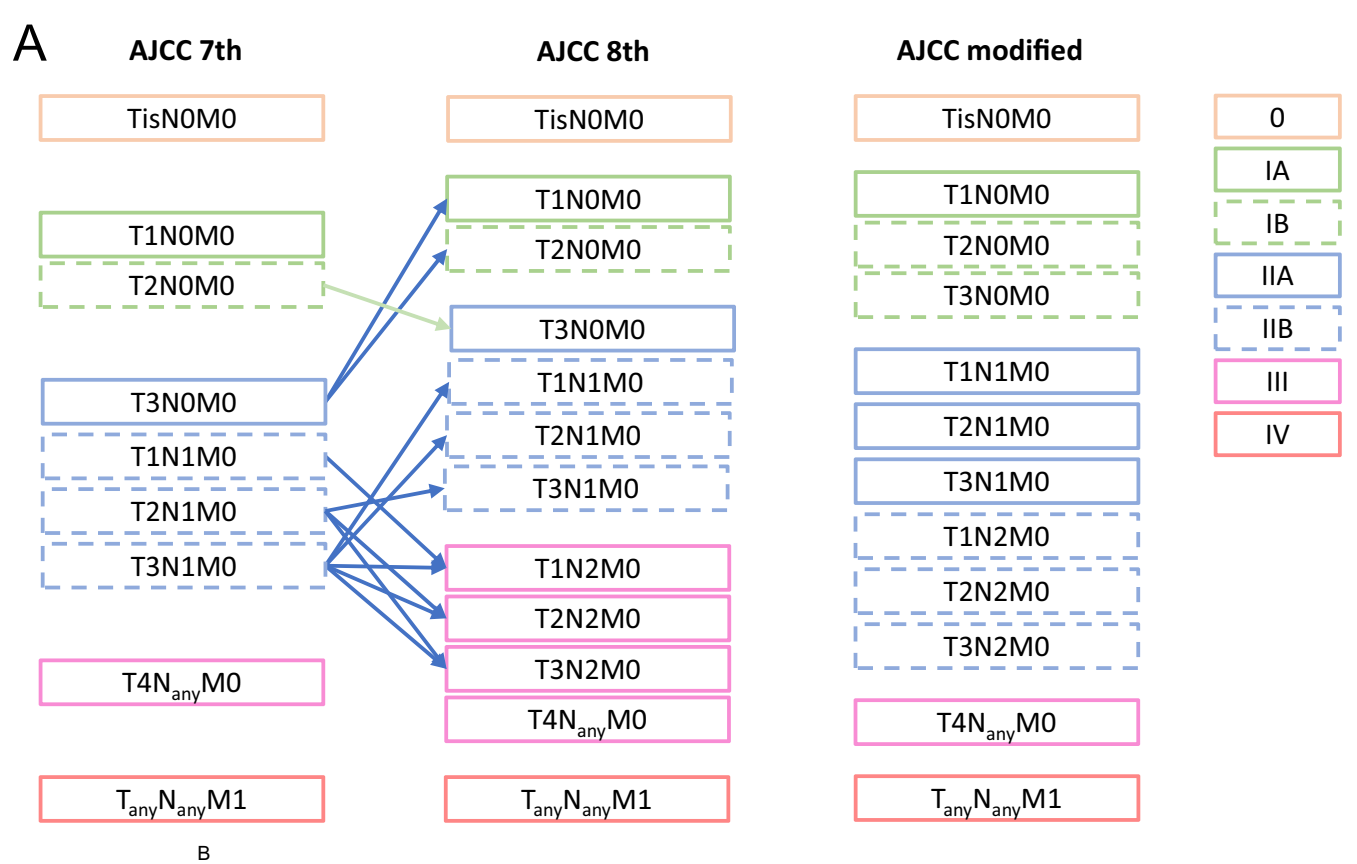

B

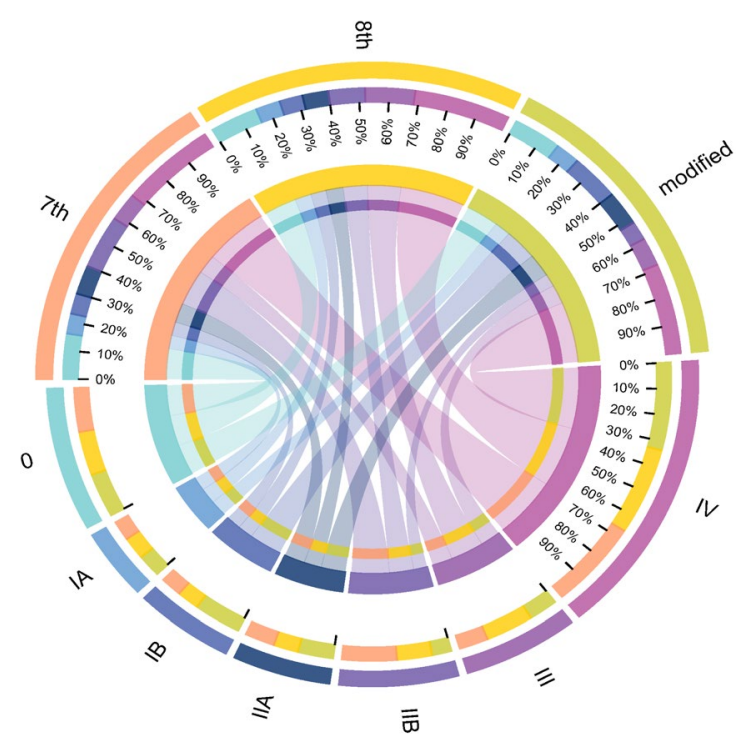

Fig. 1 Stage systems changed between the 7th, 8th and modified edition of the AJCC staging systems (A); Circos plot of the distribution difference between AJCC 7th, AJCC 8th and modified stage system in this study (B)

when using AJCC 8th, were not sufficiently distinguishable ( $p>0.05)$ (Fig. 2A, B). Similar results are shown in the Kaplan-Meier curves.

We concluded that the current AJCC 8th was not sufficiently accurate for malignant IPMN. The median OS time and univariate analysis results of patients for each substage of the AJCC 8th in the primary cohort are shown in Fig. 3. The composite measure combined these indicators, and we regrouped the substages and arrived at a modified staging system (AJCC modified) based on the median OS, pairwise comparison results, and HRs of each substage (Table 1). Although the C-index $(0.779 ; 95 \%$ CI $0.755-0.803)$ did not change significantly (Table 3 ), the pairwise comparisons of AJCC modified were all statistically significantly 
Table 2 Baseline characteristics

\begin{tabular}{|c|c|c|c|c|c|c|c|c|c|}
\hline Age, $n, \%$ & \multicolumn{2}{|c|}{$\begin{array}{l}\text { The primary cohort } \\
(n=1401)\end{array}$} & \multicolumn{2}{|c|}{$\begin{array}{l}\text { The validation cohort } \\
(n=600)\end{array}$} & & \multicolumn{2}{|c|}{$\begin{array}{l}\text { The primary cohort } \\
(n=1401)\end{array}$} & \multicolumn{2}{|c|}{$\begin{array}{l}\text { The validation } \\
\text { cohort }(n=600)\end{array}$} \\
\hline$<70$ & 800 & $57.10 \%$ & 366 & $61.0 \%$ & Yes & 646 & $46.11 \%$ & 300 & $50.0 \%$ \\
\hline$\geq 70$ & 601 & $42.90 \%$ & 234 & $39.0 \%$ & No/unknown & 755 & $53.89 \%$ & 300 & $50.0 \%$ \\
\hline Sex, n, \% & & & & & Radiotherapy, n, \% & & & & \\
\hline Female & 671 & $47.89 \%$ & 288 & $48.0 \%$ & Yes & 282 & $20.12 \%$ & 120 & $20.0 \%$ \\
\hline Male & 730 & $52.11 \%$ & 353 & $58.8 \%$ & No & 1199 & $85.58 \%$ & 480 & $80.0 \%$ \\
\hline \multicolumn{10}{|c|}{ Marital status, n, \% } \\
\hline Married & 863 & $61.60 \%$ & 353 & $58.8 \%$ & & & & & \\
\hline \multirow[t]{2}{*}{ Others ${ }^{\mathrm{a}}$} & 538 & $38.40 \%$ & 247 & $41.2 \%$ & & & & & \\
\hline & & & & & AJCC 7th, n, \% & & & & \\
\hline Race, n, \% & & & & & 0 & 222 & $15.85 \%$ & 98 & $16.3 \%$ \\
\hline Others & 151 & $10.78 \%$ & 59 & $9.8 \%$ & $\mathrm{IA}$ & 98 & $7.00 \%$ & 20 & $3.3 \%$ \\
\hline Black & 1096 & $78.23 \%$ & 475 & $79.2 \%$ & IB & 100 & $7.14 \%$ & 41 & $6.8 \%$ \\
\hline \multirow[t]{2}{*}{ White } & 154 & $10.99 \%$ & 66 & $11.0 \%$ & $\| \mathrm{A}$ & 141 & $10.06 \%$ & 66 & $11.0 \%$ \\
\hline & & $0.00 \%$ & & & $\| B$ & 260 & $18.56 \%$ & 101 & $16.8 \%$ \\
\hline Location, n, \% & & & & & III & 138 & $9.85 \%$ & 74 & $12.3 \%$ \\
\hline Head & 762 & $54.39 \%$ & 339 & $56.5 \%$ & IV & 442 & $31.55 \%$ & 200 & $33.3 \%$ \\
\hline Body or tail & 387 & $27.62 \%$ & 160 & $26.7 \%$ & & & & & \\
\hline \multirow[t]{2}{*}{ Others $^{\mathrm{b}}$} & 353 & $25.20 \%$ & 101 & $16.8 \%$ & AJCC 8th, n, \% & & & & \\
\hline & & & & & 0 & 222 & $15.85 \%$ & 98 & $16.3 \%$ \\
\hline Grade, n, \% & & & & & $\mathrm{IA}$ & 113 & $8.07 \%$ & 33 & $5.5 \%$ \\
\hline$H_{i g h}{ }^{c}$ & 191 & $13.63 \%$ & 78 & $13.0 \%$ & $\mathrm{IB}$ & 107 & $7.64 \%$ & 48 & $8.0 \%$ \\
\hline \multirow[t]{2}{*}{ Others $^{d}$} & 1210 & $86.37 \%$ & 522 & $87.0 \%$ & $\| A$ & 119 & $8.49 \%$ & 46 & $7.7 \%$ \\
\hline & & & & & $\| \mathrm{B}$ & 168 & $11.99 \%$ & 66 & $11.0 \%$ \\
\hline ELN, n, \% & & & & & III & 230 & $16.42 \%$ & 109 & $18.2 \%$ \\
\hline$<15$ & 1012 & $72.23 \%$ & 448 & $74.7 \%$ & IV & 442 & $31.55 \%$ & 200 & $33.3 \%$ \\
\hline \multirow[t]{2}{*}{$\geq 15$} & 389 & $27.77 \%$ & 152 & $25.3 \%$ & & & & & \\
\hline & & & & & \multicolumn{5}{|l|}{ AJCC modified, n, \% } \\
\hline Extend, $n, \%$ & & & & & 0 & 222 & $15.85 \%$ & 98 & $16.3 \%$ \\
\hline Inside & 666 & $47.54 \%$ & 275 & $45.8 \%$ & IA & 113 & $8.07 \%$ & 33 & $5.5 \%$ \\
\hline \multirow[t]{2}{*}{ Beyond } & 735 & $52.46 \%$ & 325 & $54.2 \%$ & IB & 226 & $16.13 \%$ & 94 & $15.7 \%$ \\
\hline & & & & & $\| \mathrm{A}$ & 168 & $11.99 \%$ & 66 & $11.0 \%$ \\
\hline Surgery, n, \% & & & & & $\| \mathrm{B}$ & 92 & $6.57 \%$ & 35 & $5.8 \%$ \\
\hline Yes & 862 & $61.53 \%$ & 343 & $57.2 \%$ & III & 138 & $9.85 \%$ & 74 & $12.3 \%$ \\
\hline No & 539 & $38.47 \%$ & 257 & $42.8 \%$ & IV & 442 & $31.55 \%$ & 200 & $33.3 \%$ \\
\hline
\end{tabular}

a "Others" in marital status include single (never married), separated, divorced, widowed, unmarried or domestic partner and unknown

b "Others" in location include pancreatic duct, others specified part of pancreas, overlapping lesion of pancreas and pancreas, NOS

c "High" in grade include poorly differentiated and undifferentiated

d "Others" in grade include well, moderately and unknown

different $(p<0.05)$. The resulting survival curves of AJCC modified are shown for the different stages in Fig. 2C.

In addition, the C-index of both AJCC (7th or 8th) and modified staging systems increased with time (Table 4).
Independent predictive factors for malignant IPMN

The results of the univariate and multivariate analyses are listed in Table 5. Multivariate analyses demonstrated that age $>70$ years, tumors located in the body and tail, high-grade differentiated tumors, surgery, chemotherapy, and tumor, lymph node, and metastasis (TNM) stages 

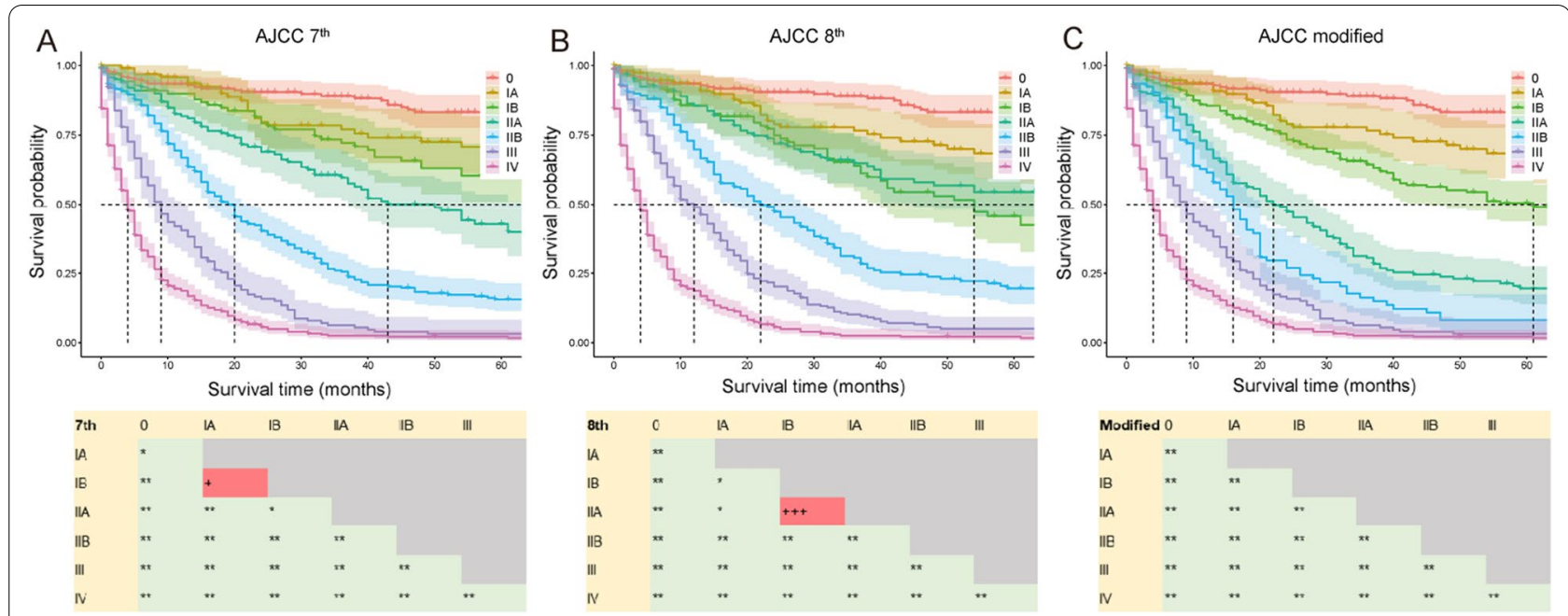

Fig. 2 Kaplan-Meier survival curves and pairwise comparison results according AJCC 7th (A), AJCC 8th (B) and AJCC modified (C) in primary cohort. Significance was determined by log-rank tests. ${ }^{*} p<0.05 ;{ }^{* *} p<0.01 ;{ }^{+} p \geq 0.05 ;{ }^{++} p \geq 0.1 ;{ }^{+++} p \geq 0.5$

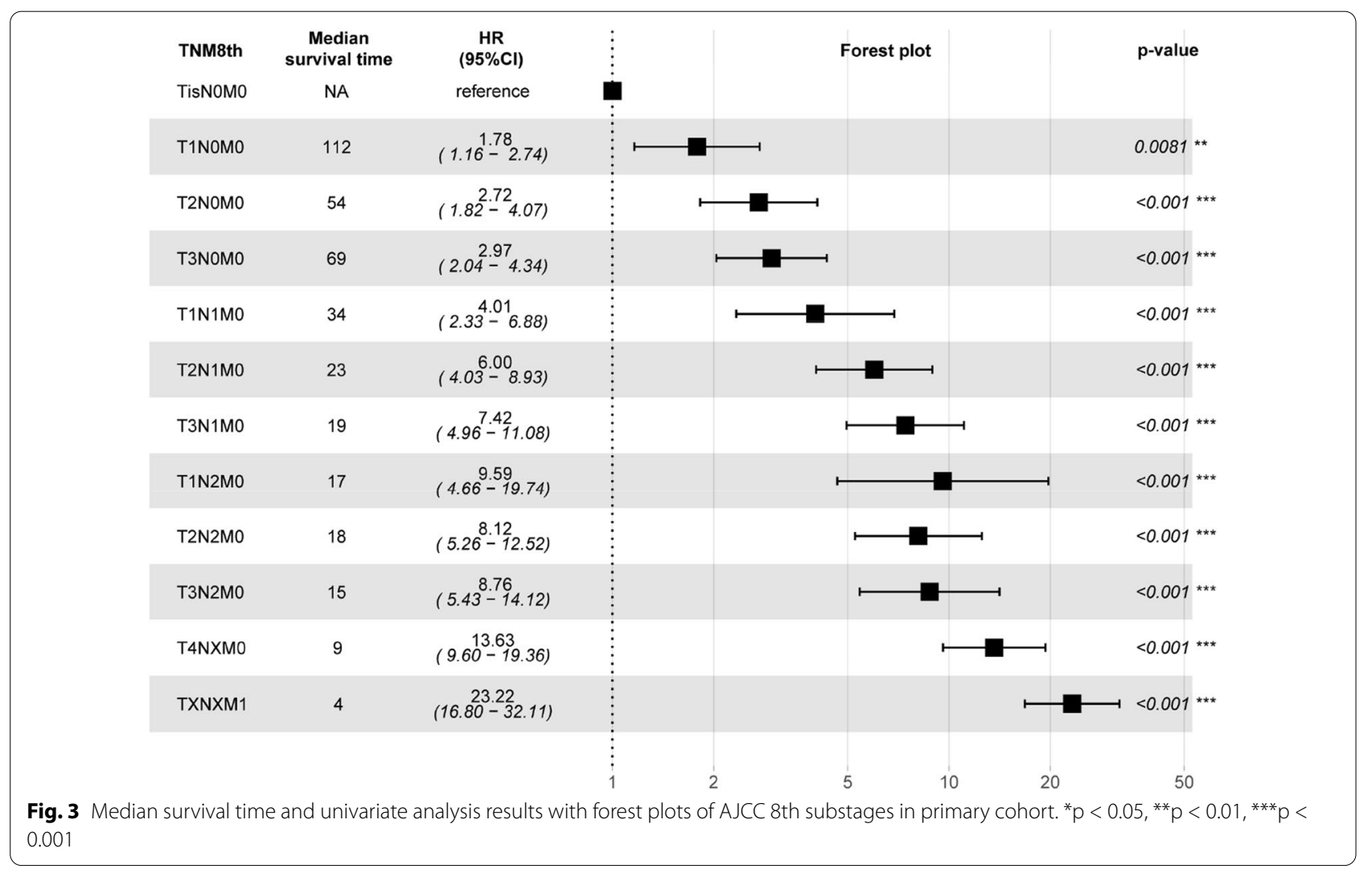

based on AJCC 8th were independent risk factors for OS $(\mathrm{p}<0.05)$.

The HRs of T2 and T3 were not significantly different (in the multivariate analysis with Tis as the reference:
T2, $\mathrm{HR}=4.97 ; 95 \%$ CI 3.50-7.04; T3, HR $=5.05 ; 95 \%$ CI 3.58-7.13). The HR of a T1a tumor was not significantly different from that of a Tis (multivariate analysis with Tis as the reference: $\mathrm{T} 1 \mathrm{a}, \mathrm{HR}=1.68$; $95 \%$ CI $0.88-3.2$; $\mathrm{p}=0.114)$. 
Table 3 Concordance indexes of different staging systems for malignant IPMN

\begin{tabular}{lll}
\hline Stage system & $\begin{array}{l}\text { The primary cohort } \\
(\mathbf{n}=\mathbf{1 4 0 1})\end{array}$ & $\begin{array}{l}\text { The validation } \\
\text { cohort }(\mathbf{n}=\mathbf{6 0 0})\end{array}$ \\
\hline AJCC 7th & $0.779(0.755-0.803)$ & $0.759(0.735-0.783)$ \\
AJCC 8th & $0.777(0.753-0.801)$ & $0.753(0.729-0.777)$ \\
AJCC modified & $0.779(0.755-0.803)$ & $0.756(0.732-0.78)$ \\
Prognostic nomogram & $0.801(0.787-0.815)$ & $0.791(0.769-0.813)$ \\
\hline
\end{tabular}

\section{Clinical predictive nomogram for OS}

The clinical predictive nomogram was developed using the predictive determinants of OS identified in the multivariate analysis (Fig. 4). The contribution of a predictor to OS can be quantified by the length of the line corresponding to each variable in the clinical predictive nomogram. We found that the T stage of AJCC 8th made the most significant contribution to survival, closely followed by surgery, the $\mathrm{N}$ stage, chemotherapy, and the $\mathrm{M}$ stage. The nomogram showed a high predictive precision, with the C-index being 0.819 (95\% CI 0.805-0.833). The 1-, 3-, 5 -year calibration curves showed a significant agreement between prediction and observation in the probability of survival (Fig. 5A-C). A similar precision was shown by the ROC curves. The values of the 1-, 3-, and 5-year OS area under the curve (AUC) were 0.881, 0.889, and 0.879, respectively (Fig. 6A-C).

\section{Validation of the clinical predictive nomogram for OS in the validation cohort}

The median age of patients at diagnosis in the validation cohort was 66 years, and the median OS of patients was 18 months (1-, 3-, 5-year survival rates: 59.4\%, 36.9\%, and $30.7 \%$, respectively).

The C-index of the established nomogram in the validation cohort was 0.791 (95\% CI 0.769-0.813). The 1-, 3-, and 5-year calibration curves (Fig. 5D-F) and the 1-, 3-, and 5-year AUC values (Fig. 6D-F) also presented ideal agreements within the primary cohort.

\section{Effect of clinical interventions on OS in the AJCC modified system}

Surgery, chemotherapy, and radiotherapy are the main clinical interventions for malignant IPMN. In the multivariate analysis, surgery and chemotherapy were statistically significantly associated with OS $(\mathrm{p}<0.05)$. To further evaluate the effect of clinical interventions in the different substages, the median OS of patients who underwent surgery and chemotherapy in each substage (I-IV) was also analyzed for the AJCC modified system. The results are presented in Fig. 7. Across all the substages (I-IV), patients who underwent surgery had a significantly longer survival time than those who did not $(\mathrm{p}<0.05, \log$-rank test). Within the II, III, and IV substages, patients who received chemotherapy had a significantly longer survival time than those who did not receive chemotherapy or when their status was not known.

\section{Discussion}

Based on the TNM system, the AJCC staging manual has become a standardized classification system for evaluating cancer at a population level in terms of the extent of disease [14]. We first evaluated the predictive value of the last two AJCC staging systems using the SEER database to assess the need for revision. The $\mathrm{C}$-index is the most widely used index to assess a model's differentiation power to correctly predicting survival. The C-indexes of the AJCC 7th and AJCC 8th in both the primary cohort ( 0.779 and 0.777 , respectively) and the validation cohort ( 0.759 and 0.753 , respectively) were not significantly different. The high predictive ability of the guideline analysis system and the significant prognostic differences in different stages can guide doctors in assessing the severity of the disease and selecting appropriate intervention. Our pairwise comparison by the log-rank test and KaplanMeier curves showed that outcomes of stage IA in AJCC 7th and stage IIA in AJCC 8th are not significantly different compared to those of stage IB. This finding indicates that the modifications from AJCC 7th to 8th did not significantly alter its clinical applicability and predictive differentiation ability, and that there are no significant differences among some of the stages in both AJCC 7th and 8th staging systems. Both systems should be further improved for malignant IPMN. Therefore, we compared the median survival time and univariate analysis results of patients in each substage of AJCC 8th and proposed a modified staging system. The modified staging system distinguished all substages sufficiently $(\mathrm{p}<0.05)$.

Table 4 Concordance indexes of different staging systems in different periods for malignant IPMN

\begin{tabular}{lcllrl}
\hline Stage system & $\mathbf{2 0 0 0 - 2 0 0 4}(\mathbf{n}=\mathbf{3 4 5})$ & $\mathbf{2 0 0 5 - 2 0 0 7}(\mathbf{n}=\mathbf{3 7 0})$ & $\mathbf{2 0 0 8 - 2 0 1 0}(\mathbf{n}=\mathbf{4 0 8})$ & $\mathbf{2 0 1 1 - 2 0 1 3}(\mathbf{n}=\mathbf{4 2 1})$ & $\mathbf{2 0 1 4 - 2 0 1 6}(\mathbf{n}=\mathbf{4 5 7})$ \\
\hline AJCC 7th & $0.688(0.673-0.703)$ & $0.773(0.761-0.785)$ & $0.763(0.749-0.777)$ & $0.78(0.766-0.794)$ & $0.807(0.791-0.823)$ \\
AJCC 8th & $0.707(0.691-0.723)$ & $0.769(0.757-0.781)$ & $0.761(0.747-0.775)$ & $0.778(0.764-0.792)$ & $0.806(0.790-0.822)$ \\
AJCC modified & $0.69(0.675-0.705)$ & $0.774(0.762-0.786)$ & $0.765(0.751-0.779)$ & $0.78(0.766-0.794)$ & $0.807(0.791-0.823)$ \\
\hline
\end{tabular}


Table 5 Univariate and multivariate analysis of factors associated with OS in the primary cohort

\begin{tabular}{|c|c|c|c|c|c|c|c|}
\hline \multirow[t]{2}{*}{ Variable } & \multirow[t]{2}{*}{ Variable levels } & \multicolumn{3}{|c|}{ Univariate analysis } & \multicolumn{3}{|c|}{ Multivariate analysis } \\
\hline & & HR & $95 \% \mathrm{Cl}$ & p-value & HR & $95 \% \mathrm{Cl}$ & $p$-value \\
\hline \multirow[t]{2}{*}{ Age } & $<70$ & Reference & & & Reference & & \\
\hline & $\geq 70$ & 1.37 & $1.21-1.56$ & $<0.001^{* * *}$ & 1.49 & $1.30-1.70$ & $<0.001^{* * *}$ \\
\hline \multirow[t]{2}{*}{ Sex } & Female & Reference & & & & & \\
\hline & Male & 0.93 & $0.82-1.05$ & 0.252 & & & \\
\hline \multirow[t]{3}{*}{ Race } & Black & Reference & & & Reference & & \\
\hline & White & 0.87 & $0.71-1.06$ & 0.163 & 1.1 & $0.9-1.36$ & 0.355 \\
\hline & Others & 0.59 & $0.44-0.78$ & $<0.001^{* * *}$ & 0.78 & $0.58-1.05$ & 0.099 \\
\hline \multirow[t]{2}{*}{ Marriage } & Others & Reference & & & Reference & & \\
\hline & Married & 0.82 & $0.72-0.94$ & $0.003^{* *}$ & 0.98 & $0.86-1.13$ & 0.801 \\
\hline \multirow[t]{3}{*}{ Location } & Head & Reference & & & Reference & & \\
\hline & Body or tail & 1.70 & $1.47-1.96$ & $<0.001^{* * *}$ & 1.22 & $1.04-1.43$ & $0.014^{*}$ \\
\hline & Others & 1.24 & $1.04-1.48$ & $0.015^{*}$ & 1.11 & $0.93-1.33$ & 0.256 \\
\hline \multirow[t]{2}{*}{ Grade } & Others & Reference & & & Reference & & \\
\hline & High & 1.38 & $1.16-1.64$ & $<0.001^{* * *}$ & 1.59 & $1.32-1.91$ & $<0.001^{* * *}$ \\
\hline \multirow[t]{2}{*}{ ELN } & $<15$ & Reference & & & Reference & & \\
\hline & $\geq 15$ & 0.51 & $0.43-0.59$ & $<0.001^{* * *}$ & 0.93 & $0.78-1.13$ & 0.479 \\
\hline \multirow[t]{2}{*}{ Extend } & Inside & Reference & & & Reference & & \\
\hline & Beyond & 2.26 & $1.97-2.58$ & $<0.001^{* * *}$ & 1.14 & $0.97-1.35$ & 0.122 \\
\hline \multirow[t]{2}{*}{ Surgery } & Yes & Reference & & & Reference & & \\
\hline & No & 5.14 & $4.48-5.90$ & $<0.001^{* * *}$ & 3.6 & $2.88-4.49$ & $<0.001^{* * *}$ \\
\hline \multirow[t]{2}{*}{ Chemotherapy } & Yes & Reference & & & Reference & & \\
\hline & No/unknown & 0.79 & $0.69-0.89$ & $<0.001^{* * *}$ & 2.04 & $1.77-2.36$ & $<0.001^{* * *}$ \\
\hline \multirow[t]{2}{*}{ Radiotherapy } & Yes & Reference & & & & & \\
\hline & No/unknown & 0.98 & $0.84-1.14$ & 0.690 & & & \\
\hline \multirow[t]{7}{*}{ T8th } & Tis & Reference & & & Reference & & \\
\hline & T1a & 1.4 & $0.74-2.66$ & 0.301 & 1.68 & $0.88-3.2$ & 0.115 \\
\hline & $\mathrm{T} 1 \mathrm{~b}$ & 1.93 & $0.91-4.11$ & 0.088 & 2.58 & $1.21-5.52$ & $0.014^{*}$ \\
\hline & T1c & 4.16 & $2.86-6.05$ & $<0.001^{* * *}$ & 3.93 & $2.66-5.79$ & $<0.001^{* * *}$ \\
\hline & $\mathrm{T} 2$ & 6.59 & $4.78-9.09$ & $<0.001^{* * *}$ & 5.13 & $3.62-7.27$ & $<0.001^{* * *}$ \\
\hline & $\mathrm{T} 3$ & 6.7 & $4.86-9.23$ & $<0.001^{* * *}$ & 5.19 & $3.68-7.33$ & $<0.001^{* * *}$ \\
\hline & $\mathrm{T} 4$ & 12.99 & $9.34-18.08$ & $<0.001^{* * *}$ & 6.16 & $4.18-9.07$ & $<0.001^{* * *}$ \\
\hline \multirow[t]{3}{*}{ N8th } & No & Reference & & & Reference & & \\
\hline & N1 & 1.21 & $1.02-1.43$ & $0.026^{*}$ & 1.55 & $1.28-1.88$ & $<0.001^{* * *}$ \\
\hline & N2 & 1.47 & $1.17-1.83$ & $<0.001^{* * *}$ & 2.94 & $2.25-3.84$ & $<0.001^{* * *}$ \\
\hline \multirow[t]{2}{*}{ M8th } & MO & Reference & & & Reference & & \\
\hline & M1 & 5.58 & $4.84-6.42$ & $<0.001^{* * *}$ & 2.35 & $1.94-2.87$ & $<0.001^{* * *}$ \\
\hline
\end{tabular}

$\mathrm{p}<0.05 ;{ }^{* *} \mathrm{p}<0.01 ;{ }^{* * *} \mathrm{p}<0.001$

Furthermore, we found that the C-indexes were increasing with time in the same evaluation system; this could be closely related to the development of medical imaging techniques [15].

Nomograms have been shown as more accurate tools than the conventional staging systems for predicting prognosis in many cancers [16-18]. Age, tumor location, differentiation grade, surgery, chemotherapy, and TNM stage in the AJCC 8th were independent factors for survival in multivariate analysis $(\mathrm{p}<0.05)$, therefore, we developed the clinical predictive nomogram. The C-index was 0.819 , which was statistically higher than that of the TNM-based stage systems (AJCC 7th, AJCC 8th and AJCC modified) in this study. Furthermore, the $1-, 3-$, and 5-AUCs of the nomogram were close to 0.90 , supporting its ability to predict individual survival accurately to a certain degree. However, our clinical predictive nomogram is more than a tool to predict survival. Furthermore, the length of the line corresponding to each variable quantifies its contribution to predicting survival. 


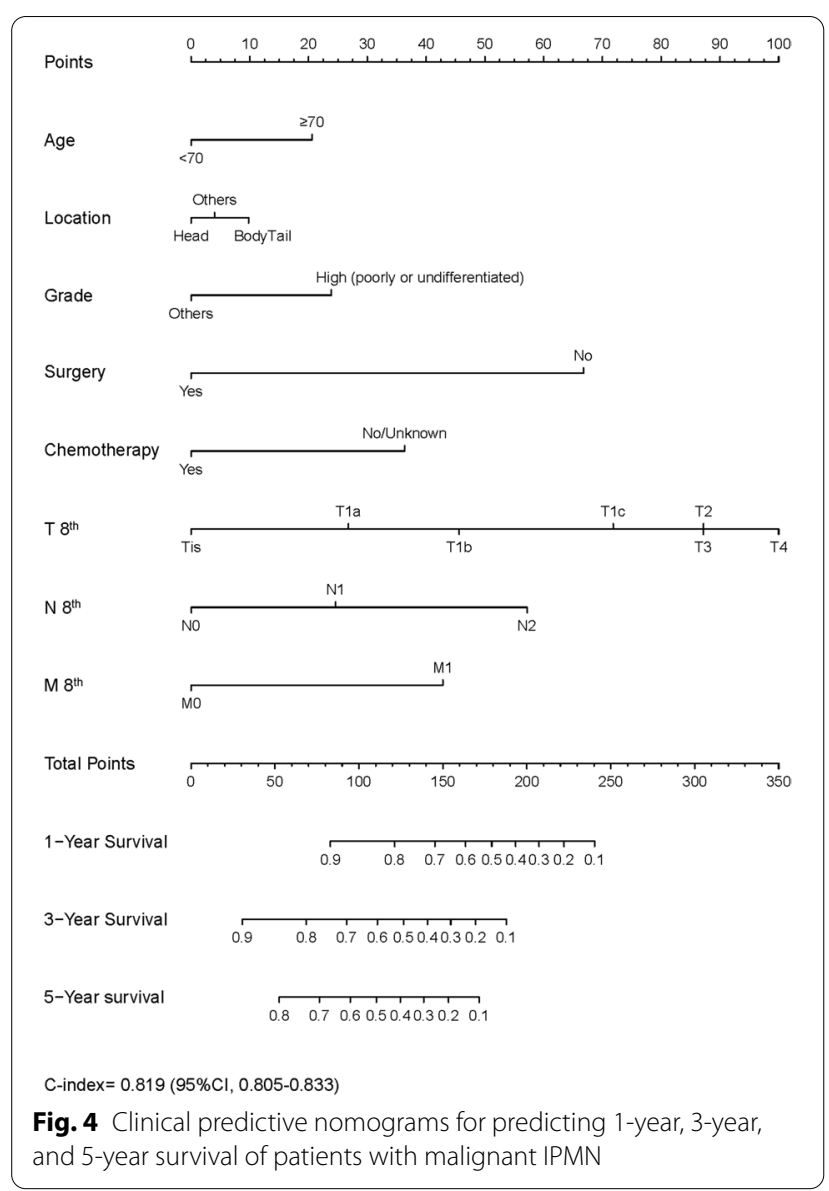

Tumor stage was the most important predictor of malignancy in malignant IPMN with the longest line in the nomogram. T-stages are mainly based on tumor size. First, we found that the HR of T1a is not significantly different from that of Tis $(\mathrm{p}<0.05)$. Invasive IPMN with tumor size $<0.5 \mathrm{~cm}$ (T1a) can be characterized as minimally invasive, which has roughly the same outcome as HGD IPMN. However, we noted that only a tumor size $<2 \mathrm{~cm}$ was an independent predictive factor; the length of the line in the nomogram and the HR of T2 and T3 were the same. The distinction of a T3 seems to be of limited predictive value, which may be the key factor affecting the accuracy of AJCC 8th for IPMN. This manifests the "degeneracy" of TMN scoring, in the condition as well as in general application in cancer, i.e. that multiple TMN scores are associated with the same stage.

As shown in previous studies, positive lymph nodes play a key role in the prognosis of IPMN $[19,20]$. The prognoses of patients with N2 tumors are significantly different from those of patients with N1 and N0 tumors, which shows that the number of positive lymph nodes is one of the independent predictive factors for malignant IPMN in our study. Significant differences can also be seen in the median survival time of patients with different $\mathrm{N}$ stages. An adequate number of examined lymph nodes (ELNs) is necessary to evaluate $\mathrm{N}$ staging. The more local lymph nodes are examined, the more accurate the $\mathrm{N}$ staging becomes. Regional lymph node metastases are frequent in patients with invasive IPMN (26.3\%, $447 / 1699$ in the entire cohort). This finding is consistent with those of previous reports $[4,21]$. Based on the above studies, lymph node dissection similar to that done for PDAC might be necessary for malignant IPMN. However, in our study, multivariate analysis showed that even a number of ELNs > 15 made no significant difference in survival.

Our results support the concept that malignant IPMN located in the pancreatic head have a better OS than those in the body or tail (head vs. body or tail, $H R=1.22$, $\mathrm{p}=0.014)$. These results support the findings of most previous studies on IPMN [22-24]. Kerlakian et al., demonstrated that jaundice was more often seen in patients with uncinate or head cysts $(14.9 \%$ vs. $1.9 \%, \mathrm{p}<0.01)$ and that incidentally discovered or asymptomatic IPMN were more likely in patients with tumors located in the neck, body, or tail of the pancreas $(53.3 \%$ vs. $31.0 \%, \mathrm{p}<0.01)$ [25]. Furthermore, the median time from diagnosis to surgery was shorter. The insidious nature of symptoms in the early stages of IPMN located in the body and tail may explain why these patients have worse outcomes. Moreover, a Japanese study showed that body or tail pancreatic IPMN is one of the independent risk factors for metachronous high-risk lesions in the remanent pancreas [21].

Surgery remains the mainstay of treatment in properly selected patients with malignant IPMN. Performing surgery resulted in significantly better survival in patients with the same stage of disease as in our study, and even in patients with distant metastasis. However, outcomes after surgical resection show that once malignant IPMN progresses to invasive, or even HGD, recurrence is common $[4,7,26]$.

The oncological benefits of adjuvant therapy for malignant IPMN remain controversial. Therefore, we deliberately included radiotherapy and chemotherapy as parameters in this study. Chemotherapy was an independent predictive factor of survival. The median survival time significantly improved for patients with stages II, III, and IV (AJCC modified), which suggests that chemotherapy may result in better survival in patients with locally advanced cancer or distant metastases. Some retrospective studies support this notion [19, 27]. Although the analysis of chemotherapy data reveals its potential in improving the prognosis of treatment. It is worth noting that SEER chemotherapy and surgery data are incomplete and may not generally be used for outcomes research 

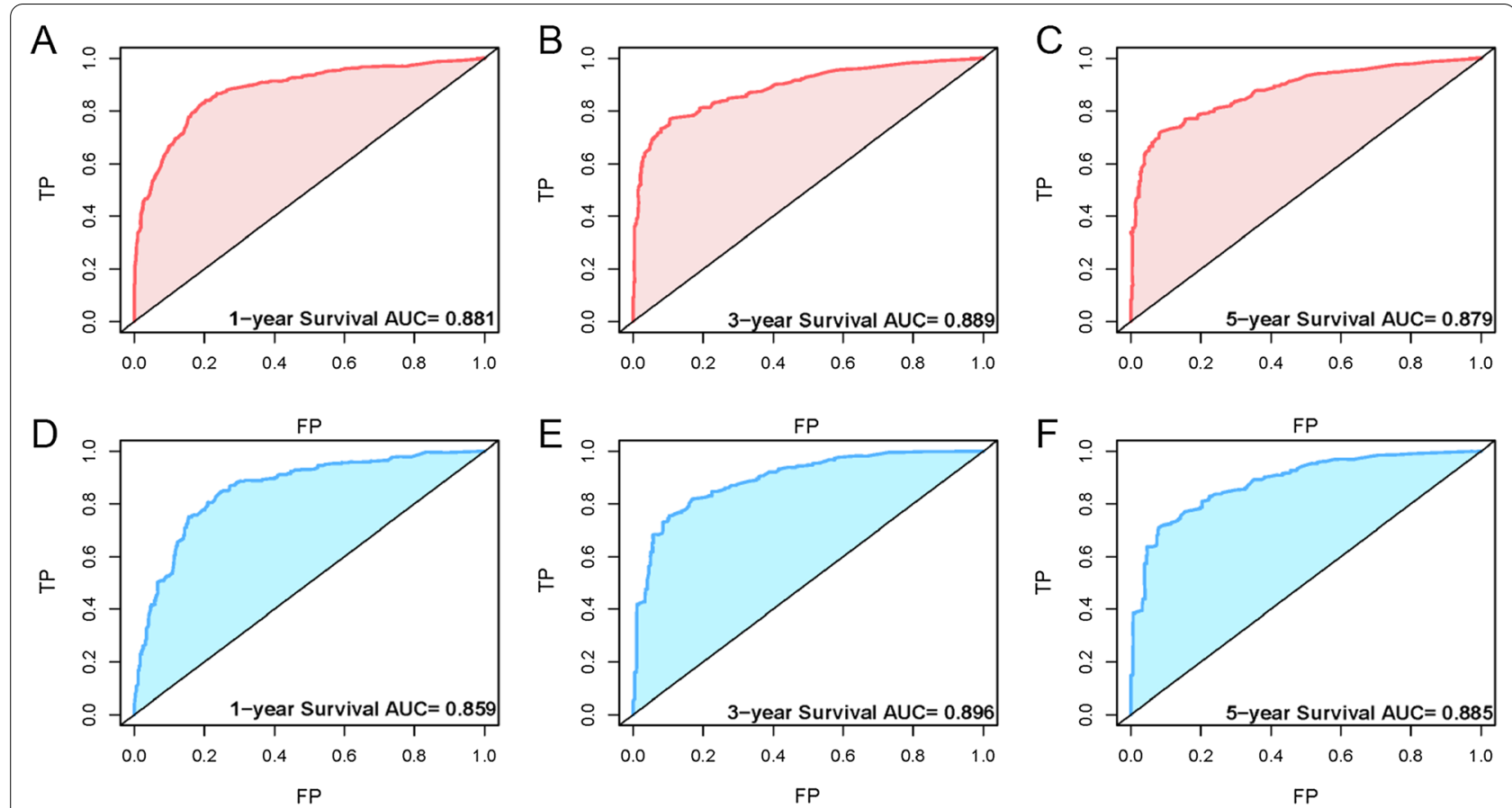

Fig. 5 The receiver operating characteristic curve of clinical predictive nomogram for predicting patient survival at 1-year (A), 3-year (B) and 5-year (C) in the primary cohort. The receiver operating characteristic curve of clinical predictive nomogram for predicting patient survival at 1-year (D), 3-year $(\mathbf{E})$ and 5-year $(\mathbf{F})$ in the validation cohort ( $F P=$ false-positive; $T P=$ true-positive)

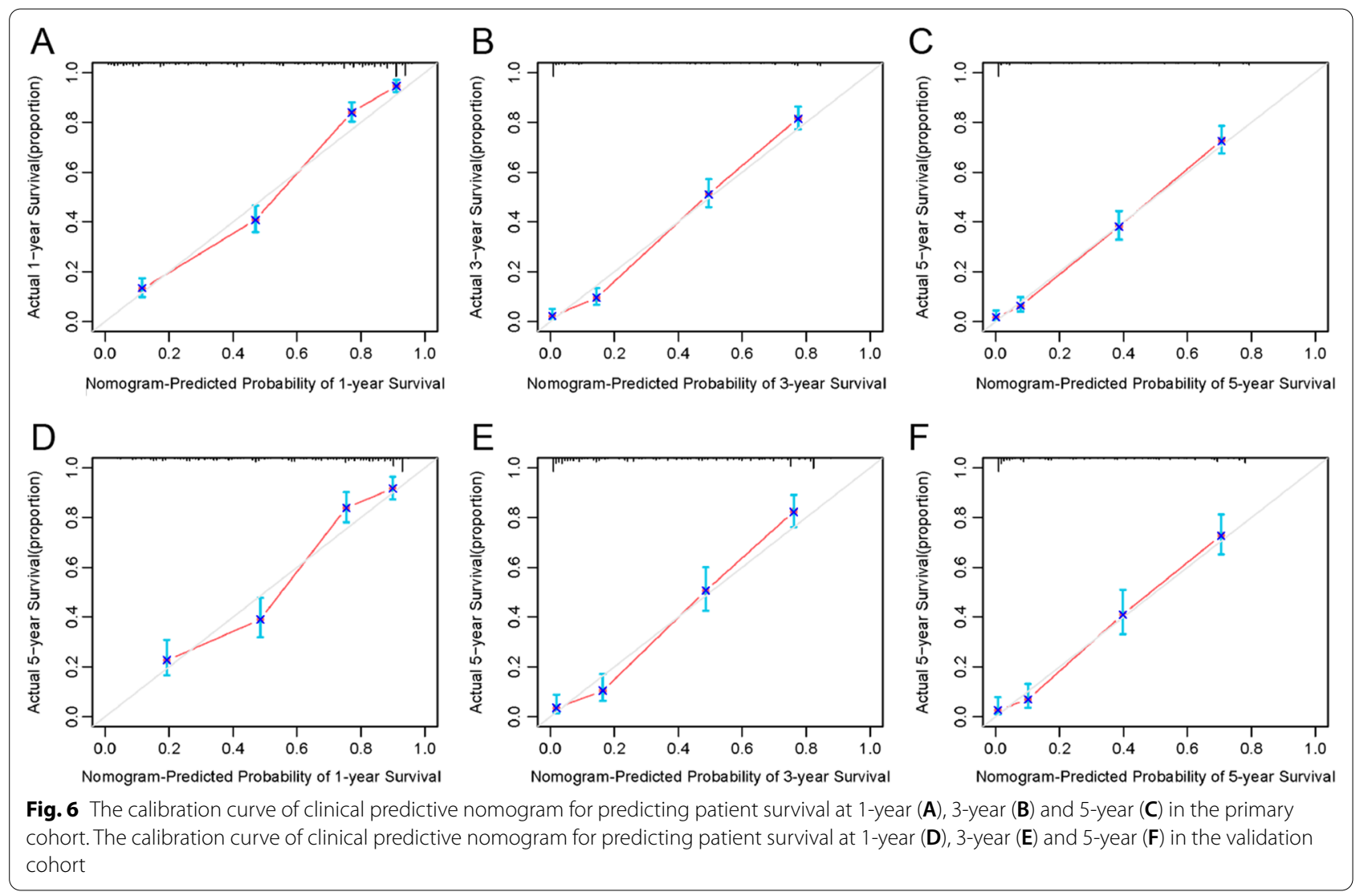




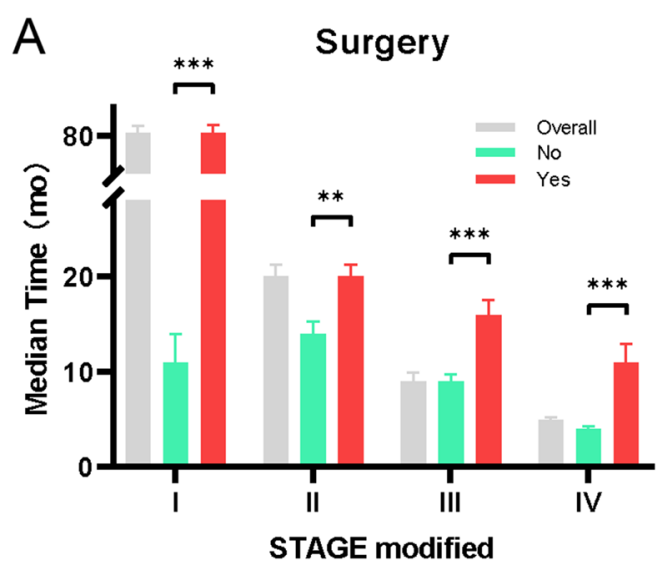

B Chemotherapy

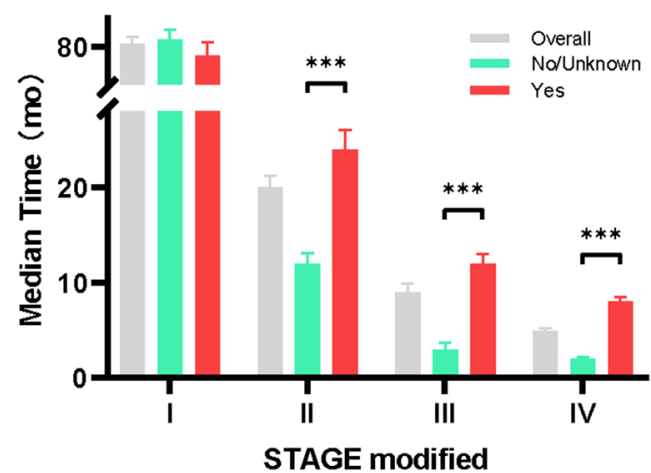

Fig. 7 The median OS with surgery (yes or no) (A), chemotherapy (yes or no/unknown) (B) in stage I, II, III and IV based on AJCC modified in the entire cohort. Significance was determined by log-rank tests. ${ }^{*} p<0.05 ;{ }^{* *} p<0.01 ;{ }^{* * *} p<0.001$

[28]. Therefore, the benefits of adjuvant therapy needs to be confirmed through large-scale studies in the future.

A long follow-up duration and a large patient population are the strengths of our study. Nevertheless, there are limitations in this study. First, it was a long-term, large-sample retrospective study; therefore, our findings need to be confirmed in a prospective cohort. With technological improvements (including diagnostic procedures and laboratory testing), different outcomes may emerge in future research. Second, although all stages were sufficiently distinguished in the modified system, the predictive ability did not significantly increase as compared to the AJCC systems; the TNM staging alone seems inadequate to further improved accurately predict the clinical outcomes of patients with malignant IPMN. Third, erroneous data or incorrect coding are still possible in the SEER database. Despite these limitations, our study of the predictive factors in malignant IPMN provides critical information for future guidelines and prospective studies.

\section{Conclusions}

We compared the accuracy of the survival prognosis of the current two AJCC guidelines and proposed a modified system to overcome their limitations. Our analysis of independent predictive factors in malignant IPMN enabled us to build an accurate and practical clinical predictive nomogram that showed a strong objective predictive power when validated. The limited predictive ability of T3 may be a key factor that affects the accuracy of AJCC 8th for malignant IPMN. Surgery remains the only potentially curative treatment and could help improve the poor prognosis of all malignant IPMN patients. For patients with locally advanced tumors or distant metastases, chemotherapy may result in better survival. Owing to the retrospective nature of our study, further prospective studies are required.

\section{Abbreviations}

AJCC: American Joint Committee on Cancer; IPMN: Intraductal papillary mucinous neoplasms; PDAC: Pancreatic ductal adenocarcinoma; HGD: Highgrade dysplasia; AJCC 8th: AJCC 8th edition staging system; AJCC 7th: AJCC 7th edition staging system; SEER: Surveillance, Epidemiology, and End Results; HRs: Hazard ratios; Cl: Confidence intervals; C-index: Concordance index; OS: Overall survival; ROC: Receiver operating characteristic; ELNs: Examined lymph nodes.

\section{Acknowledgements}

We would like to thank the National Cancer Institute for access to the database.

\section{Authors' contributions}

HXZ, CGG, and TY contributed to the conception of the study. HXZ, CGG, and TY contributed significantly to analysis and manuscript preparation. HXZ, CGG performed the data analyses and wrote the manuscript. JSC, SHW, and JWB helped perform the analysis with constructive discussions. All authors read and approved the final manuscript.

\section{Funding}

This work was supported by the National Natural Science Foundation of China (81772564).

\section{Availability of data and materials}

The datasets used and/or analyzed during the current study are available from the corresponding author on reasonable request.

\section{Declarations}

\section{Ethics approval and consent to participate}

The study design was approved by the ethics committee of Union Hospital, Tongji Medical College, Huazhong University of Science and Technology. The need for informed consent was waived owing to the study being a population study deemed not to constitute human subject research.

\section{Consent for publication}

Not applicable.

\section{Competing interests}

The authors declare that they have no competing interests. 
Received: 5 December 2020 Accepted: 8 December 2021

Published online: 24 December 2021

\section{References}

1. Rickaert F, Cremer M, Deviere J, Tavares L, Lambilliotte JP, Schroder S, et al. Intraductal mucin-hypersecreting neoplasms of the pancreas. A clinicopathologic study of eight patients. Gastroenterology. 1991;101:512-9.

2. Khan S, Sclabas G, Reid-Lombardo KM. Population-based epidemiology, risk factors and screening of intraductal papillary mucinous neoplasm patients. World J Gastrointest Surg. 2010;2:314-8.

3. Reid-Lombardo KM, St Sauver J, Li Z, Ahrens WA, Unni KK, Que FG. Incidence, prevalence, and management of intraductal papillary mucinous neoplasm in Olmsted County, Minnesota, 1984-2005: a population study. Pancreas. 2008:37:139-44

4. Oyama H, Tada M, Takagi K, Tateishi K, Hamada T, Nakai Y, et al. Longterm risk of malignancy in branch-duct intraductal papillary mucinous neoplasms. Gastroenterology. 2020;158:226-237.e225.

5. Bosman FT, World Health Organization, International Agency for Research on Cancer. WHO classification of tumours of the digestive system. 4th ed. Lyon: International Agency for Research on Cancer; 2010.

6. Tanaka M, Fernandez-Del Castillo C, Kamisawa T, Jang JY, Levy P, Ohtsuka $T$, et al. Revisions of international consensus Fukuoka guidelines for the management of IPMN of the pancreas. Pancreatology. 2017;17:738-53.

7. Rezaee N, Barbon C, Zaki A, He J, Salman B, Hruban RH, et al. Intraductal papillary mucinous neoplasm (IPMN) with high-grade dysplasia is a risk factor for the subsequent development of pancreatic ductal adenocarcinoma. HPB. 2016:18:236-46.

8. European Study Group on Cystic Tumours of the Pancreas. European evidence-based guidelines on pancreatic cystic neoplasms. Gut. 2018;67:789-804

9. Amin MB, American Joint Committee on Cancer, American Cancer Society. AJCC cancer staging manual. 8th ed. Chicago: American Joint Committee on Cancer, Springer; 2017

10. Edge SB, American Joint Committee on Cancer. AJCC cancer staging manual. 7th ed. New York: Springer; 2010.

11. Shi S, Hua J, Liang C, Meng Q, Liang D, Xu J, et al. Proposed modification of the 8th edition of the AJCC staging system for pancreatic ductal adenocarcinoma. Ann Surg. 2019;269:944-50.

12. Wasif N, Bentrem DJ, Farrell JJ, Ko CY, Hines OJ, Reber HA, et al. Invasive intraductal papillary mucinous neoplasm versus sporadic pancreatic adenocarcinoma: a stage-matched comparison of outcomes. Cancer. 2010;116:3369-77.

13. Zhou ZR, Wang WW, Li Y, Jin KR, Wang $X Y$, Wang ZW, et al. In-depth mining of clinical data: the construction of clinical prediction model with $\mathrm{R}$. Ann Transl Med. 2019;7:796.

14. Amin MB, Greene FL, Edge SB, Compton CC, Gershenwald JE, Brookland RK, et al. The eighth edition AJCC cancer staging manual: continuing to build a bridge from a population-based to a more "personalized" approach to cancer staging. CA Cancer J Clin. 2017;67:93-9.

15. Hecht EM, Khatri G, Morgan D, Kang S, Bhosale PR, Francis IR, et al. Intraductal papillary mucinous neoplasm (IPMN) of the pancreas: recommendations for standardized imaging and reporting from the society of abdominal radiology IPMN disease focused panel. Abdom Radiol. 2021;46:1586-606.

16. Balachandran VP, Gonen M, Smith JJ, DeMatteo RP. Nomograms in oncology: more than meets the eye. Lancet Oncol. 2015;16:e173-180.

17. Corso G. ASO author reflections: clinical implication of nomograms in the breast oncology field. Ann Surg Oncol. 2020;27:1875-6.

18. Wang Y, Li J, Xia Y, Gong R, Wang K, Yan Z, et al. Prognostic nomogram for intrahepatic cholangiocarcinoma after partial hepatectomy. J Clin Oncol. 2013;31:1188-95

19. McMillan MT, Lewis RS, Drebin JA, Teitelbaum UR, Lee MK, Roses RE, et al. The efficacy of adjuvant therapy for pancreatic invasive intraductal papillary mucinous neoplasm (IPMN). Cancer. 2016;122:521-33.

20. Worni M, Akushevich I, Gloor B, Scarborough J, Chino JP, Jacobs DO, et al. Adjuvant radiotherapy in the treatment of invasive intraductal papillary mucinous neoplasm of the pancreas: an analysis of the surveillance epidemiology, and end results registry. Ann Surg Oncol. 2012;19:1316-23.
21. Passot G, Lebeau R, Hervieu V, Ponchon T, Pilleul F, Adham M. Recurrences after surgical resection of intraductal papillary mucinous neoplasm of the pancreas: a single-center study of recurrence predictive factors. Pancreas. 2012;41:137-41.

22. Al Efishat M, Attiyeh MA, Eaton AA, Gonen M, Basturk O, Klimstra D, et al. Progression patterns in the remnant pancreas after resection of non-invasive or micro-invasive intraductal papillary mucinous neoplasms (IPMN). Ann Surg Oncol. 2018;25:1752-9.

23. Luo G, Fan Z, Gong Y, Jin K, Yang C, Cheng H, et al. Characteristics and outcomes of pancreatic cancer by histological subtypes. Pancreas. 2019;48:817-22.

24. Miyasaka Y, Ohtsuka T, Tamura K, Mori Y, Shindo K, Yamada D, et al. Predictive factors for the metachronous development of high-risk lesions in the remnant pancreas after partial pancreatectomy for intraductal papillary mucinous neoplasm. Ann Surg. 2016;263:1180-7.

25. Kerlakian S, Dhar VK, Abbott DE, Kooby DA, Merchant NB, Kim HJ, et al. Cyst location and presence of high grade dysplasia or invasive cancer in intraductal papillary mucinous neoplasms of the pancreas: a seven institution study from the central pancreas consortium. HPB. 2019;21:482-8.

26. Majumder S, Philip NA, Nagpal SJS, Takahashi N, Mara KC, Kendrick ML, et al. High-grade dysplasia in resected main-duct intraductal papillary mucinous neoplasm (MD-IPMN) is associated with an increased risk of subsequent pancreatic cancer. Am J Gastroenterol. 2019;114:524-9.

27. Duconseil P, Perinel J, Autret A, Adham M, Sauvanet A, Chiche L, et al. Resectable invasive IPMN versus sporadic pancreatic adenocarcinoma of the head of the pancreas: should these two different diseases receive the same treatment? A matched comparison study of the French Surgical Association (AFC). Eur J Surg Oncol. 2017:43:1704-10.

28. Noone AM, Lund JL, Mariotto A, Cronin K, McNeel T, Deapen D, et al. Comparison of SEER treatment data with medicare claims. Med Care. 2016;54:e55-64.

\section{Publisher's Note}

Springer Nature remains neutral with regard to jurisdictional claims in published maps and institutional affiliations.

Ready to submit your research? Choose BMC and benefit from

- fast, convenient online submission

- thorough peer review by experienced researchers in your field

- rapid publication on acceptance

- support for research data, including large and complex data types

- gold Open Access which fosters wider collaboration and increased citations

- maximum visibility for your research: over 100M website views per year

At BMC, research is always in progress.

Learn more biomedcentral.com/submissions 